\title{
On the Navigability of Social Tagging Systems
}

\author{
Denis Helic ${ }^{*}$, Christoph Trattner $^{\dagger}$, Markus Strohmaier* ${ }^{*}$, Keith Andrews ${ }^{\dagger}$ \\ * Knowledge Management Institute \\ Graz University of Technology \\ Graz, Austria \\ Email: \{dhelic,markus.strohmaier\}@tugraz.at \\ $\dagger$ Institute for Information Systems and Computer Media \\ Graz University of Technology \\ Graz, Austria \\ Email: $\{$ ctrattner,kandrews $\} @$ iicm.edu \\ $\ddagger$ Know-Center, Graz University of Technology, Graz, Austria
}

\begin{abstract}
It is a widely held belief among designers of social tagging systems that tag clouds represent a useful tool for navigation. This is evident in, for example, the increasing number of tagging systems offering tag clouds for navigational purposes, which hints towards an implicit assumption that tag clouds support efficient navigation. In this paper, we examine and test this assumption from a network-theoretic perspective, and show that in many cases it does not hold. We first model navigation in tagging systems as a bipartite graph of tags and resources and then simulate the navigation process in such a graph. We use network-theoretic properties to analyse the navigability of three tagging datasets with regard to different user interface restrictions imposed by tag clouds. Our results confirm that tagresource networks have efficient navigation properties in theory, but they also show that popular user interface decisions (such as "pagination" combined with reverse-chronological listing of resources) significantly impair the potential of tag clouds as a useful tool for navigation. Based on our findings, we identify a number of avenues for further research and the design of novel tag cloud construction algorithms. Our work is relevant for researchers interested in navigability of emergent hypertext structures, and for engineers seeking to improve the navigability of social tagging systems.
\end{abstract}

\section{INTRODUCTION}

In social tagging systems such as Flickr and Delicious, tag clouds have emerged as an interesting alternative to traditional forms of navigation and hypertext browsing. The basic idea is that tag clouds provide navigational clues by aggregating tags and corresponding resources from multiple sources, and by displaying them in a visually appealing fashion. Users are presented with these tag clouds as a means for exploring and navigating the resource space in social tagging systems.

While tag clouds can potentially serve different purposes, there seems to be an implicit assumption among engineers of social tagging systems that tag clouds are specifically useful to support navigation. This is evident in the large-scale adoption of tag clouds for interlinking resources in numerous systems such as Flickr, Delicious, and BibSonomy. However, this Navigability Assumption has hardly been critically reflected (with some notable exceptions, for example [1]), and has largely remained untested in the past. In this paper, we will demonstrate that the prevalent approach to tag cloud-based navigation in social tagging systems is highly problematic with regard to network-theoretic measures of navigability. In a series of experiments, we will show that the Navigability Assumption only holds in very specific settings, and for the most common scenarios, we can assert that it is wrong.

While recent research has studied navigation in social tagging systems from user interface [2], [3], [4] and networktheoretic [5] perspectives, the unique focus of this paper is the intersection of these issues. With that focus, we want to answer questions such as: How do user interface constraints of tag clouds affect the navigability of tagging systems? And how efficient is navigation via tag clouds from a network-theoretic perspective?

Particularly, we will first 1) investigate the intrinsic navigability of tagging datasets without considering user interface effects, and then 2) take pragmatic user interface constraints into account. Next, 3) we will demonstrate that for many social tagging systems, the Navigability Assumption does not hold and we will finally 4) use our findings to illuminate a path towards improving the navigability of tag clouds.

To the best of our knowledge, this paper is among the first to study what we have called the Navigability Assumption of Tag Clouds, i. e. the widely held belief that tag clouds are useful for navigating social tagging systems. One of the main results of this paper is a more critical stance towards the usefulness of tag clouds as a navigational aid in tagging systems. We argue that in order to make use of the full potential of tag clouds, new ways of thinking about tag cloud algorithms are needed.

The paper is structured as follows: In Section 2 we present our network-theoretic approach to assessing navigability of tagging systems. Section 3 describes the analyzed datasets. Section 4 presents and discusses the analysis results. Based on our findings, we call for and discuss new ideas for tag cloud algorithms in Section 5. Section 6 provides an overview of related work. Finally, Section 7 concludes the paper and presents directions for future work.

\section{Network-Theoretic Model of Navigation IN TAGGING SYSTEMS}

A tagging dataset is typically modeled as a tripartite hypergraph with $V=R \cup U \cup T$, where $R$ is the resource set, $U$ is 
the user set, and $T$ is the tag set [6], [7], [8]. An annotation of a particular resource with a particular tag produced by a particular user is a hyperedge $(r, t, u)$, connecting three nodes from these three disjoint sets.

Such a tripartite hypergraph can be mapped onto three bipartite graphs connecting users and resources, users and tags, and tags and resources. For different purposes it is often more practical to analyse one or more of these bipartite graphs. For example, in the context of ontology learning, the bipartite graph of users and tags has been shown to be an effective projection [9].

In this paper, we focus on tag-resource bipartite graphs. These graphs naturally reflect the way users are supposed to adopt tag clouds for navigating social tagging systems. For example, in many tagging systems, tag clouds are intended to be used in the following way:

1) The system presents a tag cloud to the user.

2) The user selects a tag from the tag cloud.

3) The system presents a list of resources tagged with the selected tag.

4) The user selects a resource from the list of resources.

5) The system transfers the user to the selected resource, and the process potentially starts anew.

We will study this general interaction schema and model it with a simulated user moving along the edges of the tag-resource bipartite graph and alternately visiting tag and resource nodes.

To that end, we introduce a network-theoretic approach for assessing the navigability and the efficiency of navigability in such a bipartite graph. Ever since Milgram's small world experiment [10], researchers aimed to understand "navigability" and in particular "efficient" navigation of networks (for details see Section VI). Among others, two important results stem from this line of research: (1) there exist short paths between people (nodes) in a social network and (2) people are able to navigate "efficiently" through the network having only local knowledge of the network, i.e. knowing only their personal contacts.

Kleinberg [11], [12], [13] and also independently Watts [14] formalised these properties concluding that a navigable network has a short path between all - or almost all - nodes in the network [13]. Formally, such a network has a low diameter bounded polylogarithmically, i.e. by a polynomial in $\log N$, where $N$ is the number of nodes in the network, and there exists a giant component, i.e. a strongly connected component containing almost all nodes [13]. Additionally, an "efficiently" navigable network possesses certain structural properties so that it is possible to design efficient decentralised search algorithms (algorithms that only have local knowledge of the network) [11], [12], [13]. The delivery time (the expected number of steps to reach an arbitrary target node) of such algorithms is polylogarithmic or at most sub-linear in $N$.

User navigation in hypertext systems is naturally modeled as a decentralised search, i.e. at each particular node in the network, users select a new node having only local knowledge of the network and following the idea that the selected node would bring them closest to their destination node. We use this model to investigate the navigability of tag clouds next.

\section{EXPERIMENTAL SETUP}

In the following, we conduct experiments aiming to shed light on the navigability of tag-clouds in social tagging systems. We are particularly interested in studying how design decisions, such as what tags to include in a tag cloud or how many tags to display, effect the navigability of tag clouds. While, today, designers often base such decisions on intuition or heuristics, it is our goal to study the consequences of these decisions experimentally, i.e. by exploring their empirical effects on the network.

In our experiments, we used three datasets covering a range of different settings.

- Dataset Austria-Forum: This dataset consists of annotations from an Austrian encyclopedia called AustriaForum $^{1}$. The dataset contains 32,245 annotations and 12,837 unique resources. The system is at an early phase of adoption, i.e. not many users currently contribute new tags.

- Dataset BibSonomy: This dataset ${ }^{2}$ contains nearly all 916,495 annotations and 235,339 unique resources from a dump of BibSonomy [15] until 2009-01-01. Annotations from known spammers have been excluded from the dataset. This dataset is obtained from a more mature tagging system.

- Dataset CiteULike: This dataset contains 6,328,021 annotations and 1,697,365 unique resources and is available online $^{3}$. Again, this is a dataset acquired from a more mature tagging system.

Dataset Austria-Forum represents a tagging system at an early stage of adoption. Datasets BibSonomy and CiteULike are tagging systems which have reached a certain level of maturity (i.e. attracted a larger set of active users). While all three systems adopt tag clouds for navigational purposes, their specific approaches vary. However, because the datasets contain complete information about the tripartite graph, we can experimentally manipulate the data in a way that simulates different approaches to tag cloud construction consistently across all datasets. We will describe how we manipulate the data to simulate different user interface constraints next.

\section{A. User Interface Issues}

The first user interface restriction which we model is the size of a tag cloud, i.e. the maximal number of tags displayed in a tag cloud. While different tagging systems implement different design choices, we can simulate alternative choices across all datasets. For example, in some tagging systems the maximum number of tags in a tag cloud might be 20 , while in others it might be much larger.

Another important issue of tag clouds is the algorithm used to select the tags to display in a tag cloud. While, in theory,

\footnotetext{
${ }^{1}$ http://www.austria-lexikon.at

${ }^{2}$ http://www.kde.cs.uni-kassel.de/ws/dc09/

${ }^{3} \mathrm{http}: / /$ www.citeulike.org/faq/data.adp
} 
there are many ways to compute and visualise tag clouds [16], [17], [3], in practice many tagging systems follow a simple resource-specific, TopN algorithm. In resource-specific approaches to tag cloud construction, only tags assigned to the corresponding resources are considered. In TopN approaches, the top $n$ tags with the highest resource-specific frequency are chosen for display in the corresponding tag cloud. In cases where less than $n$ tags per resource are available, the remaining slots are left empty.

For the experiments aiming to study the Navigational Assumption, we used the TopN algorithm (because it is the most common) to reconstruct simulated networks of resourcespecific tag clouds for our three datasets.

Popular tags in a mature tagging system can cover hundreds or even thousands of resources, which exceeds the pragmatic limits of a system's user interface. In this situation, tagging systems usually resort to limiting the set of resources being displayed for a given tag (for example, by sorting and "paginating" the list of corresponding resources). To model such limits, we introduce a pragmatic parameter, the length of the resource list being presented, and denote it henceforth with $k$.

In the majority of tagging systems, the resource lists presented after selecting a tag are usually sorted reversechronologically (the resources most recently tagged are listed first). For simplicity, in our experiments, we select the $k$ resources for $k$-limited resource lists randomly.

\section{RESULTS}

\section{A. Intrinsic navigability of tagging systems}

We start our study by analysing the navigability of tagging systems in a synthetic network-theoretic case, i.e. without taking any user interface restrictions into account. The first row in each of Tables I(a), I(b), and I(c) present the obtained results. The results show the existence of a giant component connecting almost all of the nodes (98\%), as well as the existence of a low effective diameter (less than 7 , i.e. it is less than polynomial in $\log N$, see Figure 1).

The only exception here is the Austria-Forum dataset. We speculate that the reason for that is due to the system being in an early adoption stage. While the effective diameter of the Austria-Forum dataset is larger than the one in the two other datasets (see Figure 1), it is still limited polylogarithmically, whereas the giant component contains only $77 \%$ of nodes. This result suggests that the Navigability Assumption depends on the adoption stage of the tagging system under investigation, i. e. the assumption may only hold for more mature tagging systems BibSonomy or CiteULike. We leave the issue of identifying the point in time where immature tagging systems transition to tagging systems exhibiting more useful navigational properties to future research. At this point, we simply observe that the Navigation Assumption is sensitive to the stage of adoption of a tagging system.

Result 1: The usefulness of tag clouds for navigation is sensitive to the phase of adoption of the social tagging system.

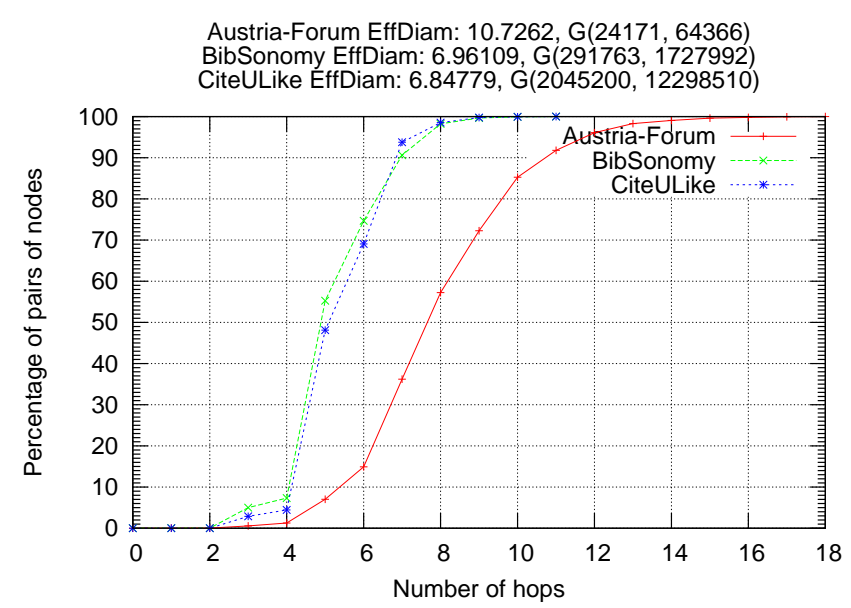

Fig. 1. Hop plots for three different tagging datasets. We can observe the shrinking diameter phenomenon [18]: The two mature datasets (Bibsonomy and CiteULike, the two lines on the left) exhibit a small diameter, while the Austria-Forum (a tagging system in an early adoption phase, the line on the right) exhibits a larger diameter, and a larger ratio of long distances between nodes.

Figures 2(a), 2(b), and 2(c) show tag (blue), resource (green), and degree (red) distributions for the analysed datasets. The tag and resource distributions were obtained by analysing a unidirectional bipartite graph, i.e. a graph with only directed links from tags to resources. The outdegree distribution and the in-degree distribution in this graph correspond to tag distribution and to resource distribution respectively. For certain ranges of degrees, both distributions are power law distributions. There are deviations in the tail of the tag distribution - these stem from the system tags assigned to imported resources (see Figures 2(b) and 2(c)). The vertical line in the tail of Figure 2(c) comes from the existence of synonym tags in the dataset. The resource distributions exhibit an exponential cut-off in the tail (see Figure 2(b)), a deviation in the tail stemming from a test resource (see Figure 2(a)), and a power law distribution as in Figure 2(c).

The degree distribution of the undirected bipartite graph (the red line in Figures 2(a), 2(b) 2(c)) combines both tag and resource distributions. For lower degrees, the combined degree distribution takes the form of the resource distribution, i.e. the number of resources with low frequencies dominates the number of tags with low frequencies. For higher degrees, the combined distribution takes the form of the tag distribution, i.e. there are more tags with high frequencies than resources with high frequencies. The tag distribution is two or more orders of magnitude larger than the resource distribution, i.e. the tag distribution strongly dominates the resource distribution for higher degrees. That means that the network hubs (high-degree nodes) are the "head" tags, i.e. the top tags for TopN tag cloud construction algorithms.

Due to the existence of a giant component and a low diameter, tagging systems are intrinsically navigable. In [19], Adamic shows the existence of efficient decentralised nav- 


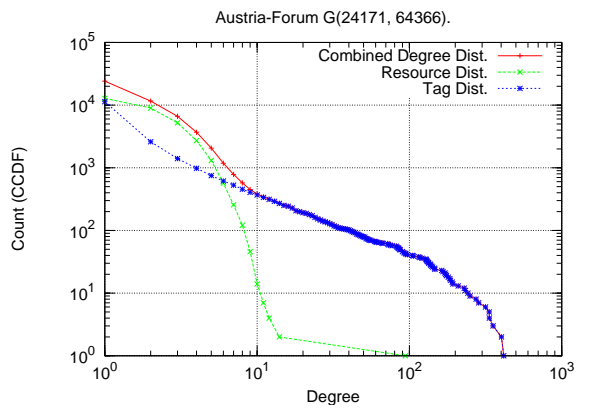

(a) Austria-Forum

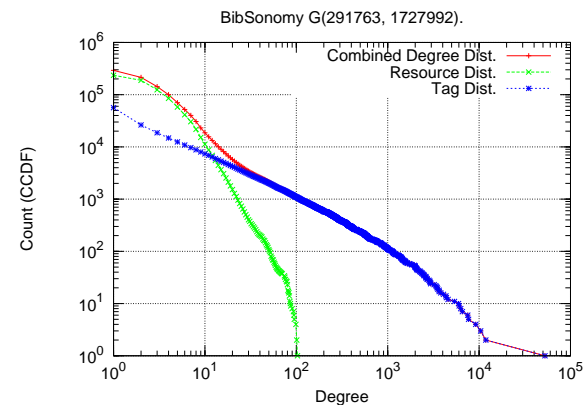

(b) BibSonomy

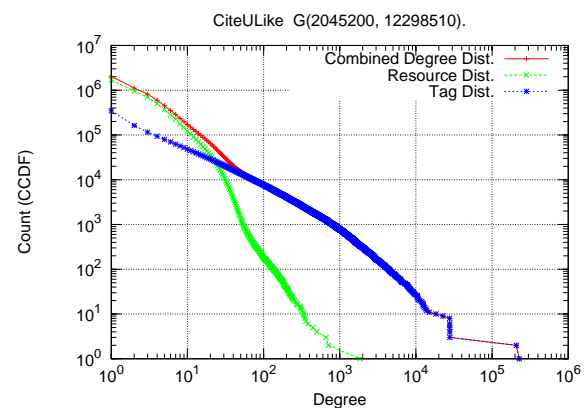

(c) CiteULike

Fig. 2. Tag, resource, and degree distributions for the three datasets.

(a) Austria-Forum

\begin{tabular}{|l|l|l|l|l|}
\hline UIR & GC & ED & UIA & NADT \\
\hline \hline none & 0.77 & 10.73 & none & sub-lin. \\
\hline \hline$n=5$ & 0.75 & 10.99 & TopN & sub-lin. \\
\hline$n=10$ & 0.76 & 11.3 & TopN & sub-lin. \\
\hline$n=20$ & 0.76 & 11.97 & TopN & sub-lin. \\
\hline$n=30$ & 0.76 & 11.05 & TopN & sub-lin. \\
\hline \hline$k=5$ & 0.36 & 12.04 & Chron. & unnav. \\
\hline$k=10$ & 0.47 & 11.16 & Chron. & unnav. \\
\hline$k=20$ & 0.56 & 10.31 & Chron. & unnav. \\
\hline$k=30$ & 0.6 & 10.68 & Chron. & unnav. \\
\hline
\end{tabular}

(b) BibSonomy

\begin{tabular}{|l|l|l|l|l|}
\hline UIR & GC & ED & UIA & NADT \\
\hline \hline none & 0.98 & 6.96 & none & sub-lin. \\
\hline \hline$n=5$ & 0.94 & 6.8 & TopN & sub-lin. \\
\hline$n=10$ & 0.97 & 6.87 & TopN & sub-lin. \\
\hline$n=20$ & 0.98 & 6.84 & TopN & sub-lin. \\
\hline$n=30$ & 0.98 & 6.91 & TopN & sub-lin. \\
\hline \hline$k=5$ & 0.31 & 6.82 & Chron. & unnav. \\
\hline$k=10$ & 0.4 & 6.62 & Chron. & unnav. \\
\hline$k=20$ & 0.5 & 6.61 & Chron. & unnav. \\
\hline$k=30$ & 0.54 & 6.65 & Chron. & unnav. \\
\hline
\end{tabular}

(c) CiteULike

\begin{tabular}{|l|c|c|l|l|}
\hline UIR & GC & ED & UIA & NADT \\
\hline \hline none & 0.98 & 6.85 & none & sub-lin. \\
\hline \hline$n=5$ & 0.93 & 6.97 & TopN & sub-lin. \\
\hline$n=10$ & 0.95 & 7.07 & TopN & sub-lin. \\
\hline$n=20$ & 0.97 & 7.17 & TopN & sub-lin. \\
\hline$n=30$ & 0.97 & 6.98 & TopN & sub-lin. \\
\hline \hline$k=5$ & 0.27 & 6.89 & Chron. & unnav. \\
\hline$k=10$ & 0.36 & 6.95 & Chron. & unnav. \\
\hline$k=20$ & 0.44 & 6.91 & Chron. & unnav. \\
\hline$k=30$ & 0.48 & 7.05 & Chron. & unnav. \\
\hline
\end{tabular}

UIR = UI Restriction, GC = Giant Component, ED = Effective Diameter, UIA = UI Algorithm, NADT = Navigation Algorithm Delivery Time Chron. $=$ Chronological algorithm, sub-lin. $=$ sub-linear, unnav. $=$ unnavigable network

TABLE I

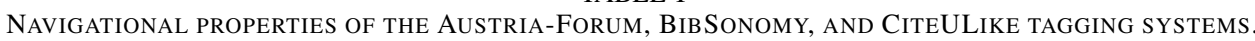

igation and search algorithms for power law networks. In principle, a user could first navigate to a hub (which is typically achieved in a few hops in a power law network) and since hubs have a large out-degree, one can reach the destination node easily. The delivery time of the algorithm is sub-linear, although the number of inspected nodes in the worst-case is $O(N)$, since sometimes the user needs to inspect all outgoing links from a hub.

Result 2: Tagging networks are navigable power-law networks. For power law networks, efficient sub-linear decentralised navigation algorithms exist.

\section{B. Tag cloud size}

Rows two to five of Tables I(a), I(b), and I(c) show the results of applying the TopN algorithm to limit the tag cloud size on the analysed datasets. From a network-theoretic point of view, limiting the tag cloud size means limiting the outdegree of the resource nodes in the bipartite graph. The outdegree of the resource nodes is two orders of magnitude smaller then the out-degree of the tag nodes, indicating there are no resource "hubs" in the network. Therefore, limiting the tag cloud size does not influence the network to a large extent. In other words, the structure of the network is still maintained, i.e. the network remains a navigable network with navigation efficiency inherent to power law networks.
Result 3: Limiting the tag cloud size to practically feasible sizes (e.g. 5, 10, or more) does not influence navigability.

\section{Pagination}

Rows six to nine of Tables I(a), I(b), and I(c) contain the results of simulating pagination with resource lists sorted reverse-chronologically. Even without experiments, it is evident that limiting the number of links going out from a tag node has destructive effects on the resulting network. In other words, limiting the out-degree of hub nodes in a power-law network destroys the connectivity of the network as a whole. Our experiments show exactly that: the giant component collapses, and the largest strongly connected component now only contains around 50\% or less nodes. As such, pagination destroys network navigability, and the Navigability Assumption only holds when we assume that users would be able and willing to inspect long lists $(>10.000)$ of resources per tag, which is not reasonable. For example, we know from search query $\log$ research that users rarely click on links beyond the first result page [20]. This yields our final result:

Result 4: Limiting the out-degree of high frequency tags (e.g. through pagination with resource lists sorted reversechronologically) leaves the network vulnerable to fragmentation. This destroys navigability of prevalent approaches to tag clouds. 
1: Input: $G=<V, E>, r, t, k$

2: for all $r \in R_{t}$ do

add $t_{r}$ to $V$

add $\left(r, t_{r}\right)$ to $E$

$R L_{t} \leftarrow f(r, k)$

for all $r r \in R L_{t}$ do

add $\left(t_{r}, r r\right)$ to $E$

end for

end for

: remove $t$ from $V$

Fig. 3. Generalized pagination algorithm

\section{IMPLICATIONS}

The previous analysis illustrated the vulnerability of tagging networks to the pagination effect, where a limit is placed on the number of links going out from paginated tags, i.e. tags with frequency higher than the pagination parameter $k$. This vulnerability is mainly due to the simplicity of the common pagination algorithm, i.e. the resource list is simply sorted reverse-chronologically and only the $k$ most recently tagged resources are presented to the user. The algorithm does not take into account the current user context, i.e. the resource where the user clicks on a paginated tag. Rather the same reverse-chronologically resource list is presented for a given paginated tag throughout the system.

Let us now investigate possibilities to recover the navigability of tagging networks by means of alternative tag construction algorithms. To this end, we introduce an adapted pagination algorithm. A simple generalisation of the pagination algorithm is to select $k$ different resources out of all resources tagged with a given paginated tag, depending on the current user context, i.e. depending on the resource where the user activates a paginated tag. Let us denote the resources list of a given paginated tag $t$ with $R_{t}$. In this case, a particular selection of resources for $t$ becomes a function of a given resource and parameter $k$, i.e. $R L_{t}=f(r, k)$. In other words, each paginated tag is replaced by as many resource-specific tags $\left(t_{r}\right)$ as there are resources in its resource list. Each resource-specific tag is then connected to resources computed by $f(r, k)$. The pseudo-code of the generalised algorithm is given in Figure 3.

We now discuss some potential functions $f(r, k)$ for selecting resources from the available resource pool and analyse their influence on network navigability.

\section{A. Random link selection}

A first obvious choice for $f(r, k)$ is to select $k$ resources uniformly at random. This approach generates a random graph as introduced by [21] for each given paginated tag. As [22] and [23] showed, graphs generated uniformly at random are typically connected and have - with a high probability - a diameter bound by $\log N$ (already for out-degrees $k \geq 3$ ). However, since there are no structural clues in a randomly generated network, a decentralized search algorithm will need to inspect, in the worst case, all nodes of the network in order to reach a destination node from the given starting node.

Table II shows the results of a random pagination algorithm on the three test datasets. All three networks become strongly connected with a giant component even for low values of $k$. As expected, all three networks also possess a low diameter.

\section{B. Hierarchical network model}

In [13], Kleinberg introduced the hierarchical network model and elegantly proved that it is possible to design efficient decentralised search algorithms for such networks with a delivery time polynomial in $\log N$ (for details see Section VI). Put simply, Kleinberg showed that, if the nodes of a network can be organised into a hierarchy, then such a hierarchy provides a probability distribution for connecting the nodes in the network. The resulting network is efficiently navigable. A special case of the hierarchical network model is given when there is a constant number of links leaving a node, i.e. when the out-degree of a node is limited by a parameter $k$ as it is the case with pagination. In this case, the tree leaves contain so-called clusters of nodes, i.e. a collection of a certain constant number of nodes.

Thus, we developed a hierarchical network generator that 1) sorts the resource list of a given paginated tag by frequency, 2) creates resource clusters of size 10 by traversing the sorted resource list sequentially, 3$)$ creates a balanced $b$-ary $(b=5)$ tree where the number of leaves is equal to the number of the resource clusters, 4) traverses the tree in postorder from left to right and attaches resource clusters to the tree leaves, and 5) uses this tree structure to obtain the link probability distribution for connecting a resource-specific tag node with resources of a given paginated tag.

It is important to note that the tree creation process follows the statistical properties of the tagging dataset only, it has no inherent semantic rationale. As such, it serves primarily as a statistical tool to improve the efficiency of navigability from a network-theoretic perspective. Table III provides an overview of the results of the structural network analysis performed with the three real-life datasets.

Another important observation is that in our model each paginated tag is a source of a network generated by a hierarchy. These networks are themselves connected through tag co-occurrence in the dataset, i.e. since tags overlap and share resources such shared resources link different generated networks. This makes it more difficult to estimate the delivery time of a decentralised search algorithm possessing only the local knowledge. If the algorithm is extended to have knowledge of all the hierarchies used in the generation of the networks, then this additional information might be useful in finding a destination node faster.

However, more theoretical work is needed to offer a proof of this intuitive assumption. In addition, it would be interesting to test these ideas empirically, for example, by implementing the algorithm and applying it to the real-life datasets. Another interesting problem is the fitting of parameters for the hierarchical network model, for example what is the optimal 
(a) Austria-Forum

\begin{tabular}{|l|l|l|l|l|}
\hline UIR & GC & ED & UIA & NADT \\
\hline \hline$k=5$ & 0.86 & 11.7 & Random & linear \\
\hline$k=10$ & 0.86 & 11.02 & Random & linear \\
\hline$k=20$ & 0.85 & 10 & Random & linear \\
\hline$k=30$ & 0.84 & 10.42 & Random & linear \\
\hline
\end{tabular}

$\mathrm{UIR}=\mathrm{UI}$ Restriction, $\mathrm{GC}=$ Giant Component (b) BibSonomy

\begin{tabular}{|l|l|l|l|l|}
\hline UIR & GC & ED & UIA & NADT \\
\hline \hline$k=5$ & 0.99 & 8.75 & Random & linear \\
\hline$k=10$ & 0.99 & 6.97 & Random & linear \\
\hline$k=20$ & 0.99 & 6.75 & Random & linear \\
\hline$k=30$ & 0.99 & 6.46 & Random & linear \\
\hline
\end{tabular}

(c) CiteULike

\begin{tabular}{|l|l|l|l|l|}
\hline UIR & GC & ED & UIA & NADT \\
\hline \hline$k=5$ & 0.99 & 7.98 & Random & linear \\
\hline$k=10$ & 0.99 & 7.88 & Random & linear \\
\hline$k=20$ & 0.99 & 7.13 & Random & linear \\
\hline$k=30$ & 0.99 & 6.86 & Random & linear \\
\hline
\end{tabular}

NADT $=$ Navigation Algorithm Delivery Time

TABLE II

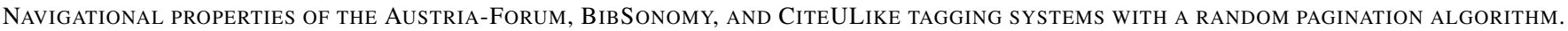

combination of the cluster size and the maximum number of children, with respect to the size of the resource list and the pagination parameter $k$.

\section{Navigational and semantic penalty}

The previous section shows that one way of designing an efficiently navigable network in a tagging system is to classify the resources of a given paginated tag into a hierarchy.

The hierarchy used in our experiments does not possess any semantic grounding, but it is optimal from a navigational point of view. However, improvements of our algorithms will need to take the semantics of the dataset into account by identifying a set of resource attributes. For example, resource attributes might be the date of creation, authors, other tags, or even attributes external to the system such as URLs, fulltext, or title. To design a navigable network, the pagination algorithm needs to organise these resource attributes into a hierarchy. At the same time, it is difficult to expect that an algorithm taking into account the semantics of resources can produce an optimal hierarchy that optimizes navigability of the tagging system as a whole. Rather, the semantic algorithm will tend to produce an unbalanced tree with a variable cluster size. As a consequence, the navigational structure generated by such an algorithm will be sub-optimal, i.e. a decentralised search algorithm will need to take more steps (investigate more nodes) to find a destination node. We will call this effect the navigational penalty. Of course, the pagination algorithm might be altered to produce a tree closer to the optimal tree from the navigational point of view. This, however, seems possible only by breaking semantics to a certain extent. We will call this contrasting effect the semantic penalty. This reveals an essential trade-off which tag cloud construction algorithms will need to address: balancing the navigational and semantic penalties.

Let us illustrate the navigational and semantic penalties with an example. Suppose we have 1000 resources about Austrian cities tagged with "Austria". A particular tagging system might decide to paginate that tag with a pagination parameter of $k=20$ (listing 20 resources per page). Firstly, the system would need to semantically classify the resources into a clustered hierarchy. For example, it could take geography as the criteria for creating clusters: each cluster corresponding to an Austrian province. However, the size of the clusters varies and the province of Vienna (the capital of Austria) might dominate, since it contains, say, 500 resources. Generating the network from such an unbalanced hierarchy will result in a navigational penalty, whereas a new classification of the resources taking into account the Vienna districts as a further geographical refinement to balance the cluster size may cause a semantic penalty, if the Vienna province is represented at a finer level of detail than other provinces.

Further research needs to shed light on how to measure and balance the trade-off between navigational and semantic penalties. For example, there are measures such as FolkRank [24] or TagCont [25] which might represent an interesting starting point for quantifying the effects of the semantic penalty.

\section{RELATED WORK}

We start our review of related work with a brief overview of network-related research. Research on network navigability has been inspired by Milgram's small world experiment [10]. In this experiment, selected persons from Nebraska received a letter they were then asked to send through their social networks to a stockbroker in Boston. The striking result of the study was that, for those letters reaching the destination, the average number of hops was around 6, i.e. the population of the USA constituted a "small world". While the conclusions have been challenged [26], this experiment has attracted a great deal of interest in the research community.

Numerous researchers analysed Milgram's experiment trying to create network models and generators able to produce such "small world" networks (see for example [27]). The lattice model by Watts [28] mimics a real-life social network, where people are primarily connected to their neighbours with a few "long-range" contacts. The networks generated by this model have, like the random graph model [22], [23], a giant component and a diameter bound by $\log N$.

Kleinberg analysed the second result of the Milgram's experiment, the ability of people to find a short path when there is such a path between two nodes [11], [12], [13]. He concluded that there are structural clues in such networks, which allow people to find a short path efficiently and argued that for an "efficiently" navigable network there exists a decentralised search algorithm with delivery time polynomial in $\log N$.

Kleinberg also designed a number of network models such as 2D-grid models [12], hierarchical models [13], and group models [13], and showed that for certain combinations of parameters, efficient decentralised search algorithms exist. 
(a) Austria-Forum

\begin{tabular}{|l|l|l|l|l|}
\hline UIR & GC & ED & UIA & NADT \\
\hline \hline$k=5$ & 0.85 & 12.03 & Hier. & polylog. \\
\hline$k=10$ & 0.86 & 10.62 & Hier. & polylog. \\
\hline$k=20$ & 0.85 & 9.29 & Hier. & polylog. \\
\hline$k=30$ & 0.84 & 9.71 & Hier. & polylog. \\
\hline
\end{tabular}

\begin{tabular}{ll|l|l|l}
$k=30$ & 0.99 & 6.75 & Hier & polylog. \\
\hline
\end{tabular}

\begin{tabular}{|l|l|l|l|l|}
\hline UIR & GC & ED & UIA & NADT \\
\hline \hline$k=5$ & 0.99 & 8.82 & Hier. & polylog. \\
\hline$k=10$ & 0.99 & 7.62 & Hier. & polylog. \\
\hline$k=20$ & 0.99 & 6.94 & Hier. & polylog. \\
\hline$k=30$ & 0.99 & 6.75 & Hier. & polylog. \\
\hline
\end{tabular}

Hier. = Hierarchical Algorithm, polylog. = polylogarithmic

TABLE III

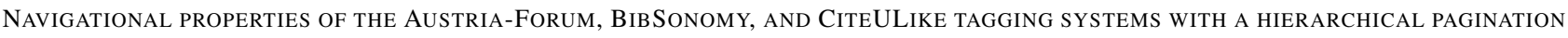
ALGORITHM.
Kleineberg also showed that there is no such algorithm for the lattice model.

Particularly, hierarchical network models [13] are based on the idea that, in many settings, the nodes in a network might be classified according to a taxonomy. The taxonomy can be represented as a $b$-ary tree and network nodes can be attached to the leaves of the tree. For each node $v$, we can create a link to all other nodes $w$ with the probability that decreases with $h(v, w)$ where $h$ is the height of the least common ancestor of $v$ and $w$ in the tree. For a constant out-degree, the nodes are clustered and then the clusters are attached to the tree. The link distribution defined by $f(h)=(h+1)^{-2} b^{-h}$ generates a navigable network with a decentralised search algorithm with delivery time of $O\left(\log _{b}^{4} N\right)$.

In related research of tagging systems, tag clouds have been characterised as a way to translate the emergent vocabulary of a folksonomy into social navigation tools [4], [29]. Social navigation itself represents a multi-dimensional concept, covering a range of different issues and ideas. A distinction between direct and indirect social navigation, for example, highlights whether navigational clues are provided by direct communication among users (e.g. via chat), or whether navigational clues are indirectly inferred from historical traces left by others [30]. Based on this distinction, our work only focuses on indirect social navigation in the sense that it studies the effectiveness of traces ("tags") left by users in tagging systems. Other types of social navigation emphasise the need to show the presence of others users, to build trust among groups of users, or to encourage certain behaviour [30].

Researchers have discussed the advantages and drawbacks of tag clouds, suggesting that tag clouds are a useful mechanism when users' search tasks are general and explorative (for example, learn about Web 2.0), while tag clouds provide little value for specific information-seeking tasks (for example, navigate to www.cnn.com) [4], [31]. While the paper at hand focuses on network-theoretic aspects, cognitive aspects of navigation have been studied previously using, for example, SNIFACT [32] and social information foraging theory [33]. Other work has studied the motivations of users for tagging [34], [35], and how they influence emergent semantic (as opposed to navigational) structures. The navigational utility of single tags has been investigated [36] with somewhat disappointing results. With time the tags become harder and harder to use as they lose specificity and reference too many resources. Such tags are exactly those paginated tags where new pagination algorithms are needed.

Navigation models for tagging systems have been also discussed recently. In [8] authors describe a navigation framework for tagging systems. The authors apply the framework to analyze possible attacks on tagging systems. In principle, the framework identifies a navigation channels as any combination of the basic elements of a tagging system (users, tags, and resources). Thus, the specific combination which we investigated in this paper can be summarized as the resource-tag or tag-resource navigation channel.

Recent literature also discusses algorithms for the construction of tag clouds. The ELSABer algorithm [37] represents an example of such an effort aimed towards identifying hierarchical relationships between annotations to facilitate browsing. The work by [38] is another example, introducing entropy-based algorithms for the construction of interesting tag clouds. However, these algorithms have not found widespread adoption in current social tagging systems. In addition, empirical studies of tagging systems have for example focused on comparing navigational characteristics of tag distributions to similar distributions produced by library terms [39].

Our work contributes to an increased theoretical understanding about the navigability of current tag cloud algorithms in social tagging systems. Our experiments identify empirical problems related to the navigability of tag clouds in three realworld tagging systems.

\section{CONCLUSION}

The motivation for this research was to examine and test the widely held belief that tag clouds support efficient navigation in social tagging systems. We have shown that for certain specific, but popular, tag cloud scenarios, the so-called Navigability Assumption does not hold. The results presented in this paper make a theoretical and an empirical argument against existing approaches to tag cloud construction. Our work thereby both confirms and refutes the assumption that current tag cloud incarnations are a useful tool for navigating social tagging systems. While we confirm that tagresource networks have efficient navigational properties in theory, we show that popular user interface decisions (such as "pagination" combined with reverse-chronological listing of resources) significantly impair navigability. Our experimental 
results demonstrate that popular approaches to using tag clouds for navigational purposes suffer from significant problems.

Building on recent research results from network theory, in particular hierarchical network models, we have illustrated a path towards constructing more efficiently navigable tag cloud networks, which are less vulnerable to pagination influences. Our findings suggest that engineers who want to design effective tag cloud algorithms have to essentially strike a balance between semantic and navigation penalties, in order to make navigation in social tagging systems both efficient and effective. We conclude that in order to make full use of the potential of tag clouds for navigating social tagging systems, new and more sophisticated ways of thinking about designing tag cloud algorithms are needed.

\section{REFERENCES}

[1] M. A. Hearst and D. Rosner, "Tag clouds: Data analysis tool or social signaller?' in HICSS '08: Proceedings of the Proceedings of the 4lst Annual Hawaii International Conference on System Sciences. Washington, DC, USA: IEEE Computer Society, 2008.

[2] C. S. Mesnage and M. J. Carman, "Tag navigation," in SoSEA '09: Proceedings of the 2nd international workshop on Social software engineering and applications. New York, NY, USA: ACM, 2009, pp. 29-32.

[3] A. W. Rivadeneira, D. M. Gruen, M. J. Muller, and D. R. Millen, "Getting our head in the clouds: toward evaluation studies of tagclouds," in CHI '07: Proceedings of the SIGCHI conference on Human factors in computing systems. New York, NY, USA: ACM, 2007, pp. 995-998.

[4] J. Sinclair and M. Cardew-Hall, "The folksonomy tag cloud: when is it useful?" Journal of Information Science, vol. 34, p. 15, 2008. [Online]. Available: http://jis.sagepub.com/cgi/content/abstract/34/1/15

[5] N. Neubauer and K. Obermayer, "Hyperincident connected components of tagging networks," in HT '09: Proceedings of the 20th ACM conference on Hypertext and hypermedia. New York, NY, USA: ACM, 2009, pp. 229-238.

[6] C. Cattuto, C. Schmitz, A. Baldassarri, V. D. P. Servedio, V. Loreto, A. Hotho, M. Grahl, and G. Stumme, "Network properties of folksonomies," AI Commun., vol. 20, no. 4, pp. 245-262, 2007.

[7] C. Schmitz, A. Hotho, R. Jäschke, and G. Stumme, "Mining association rules in folksonomies," in Data Science and Classification: Proc. of the 10th IFCS Conf., Studies in Classification, Data Analysis, and Knowledge Organization. Springer, 2006, pp. 261-270.

[8] M. Ramezani, J. Sandvig, T. Schimoler, J. Gemmell, B. Mobasher, and R. Burke, "Evaluating the impact of attacks in collaborative tagging environments," in Computational Science and Engineering, 2009. CSE '09. International Conference on, vol. 4, aug. 2009, pp. 136 -143.

[9] P. Mika, "Ontologies are us: A unified model of social networks and semantics," Web Semantics: Science, Services and Agents on the World Wide Web, vol. 5, no. 1, pp. 5-15, 2007.

[10] S. Milgram, "The small world problem," Psychology Today, vol. 1, pp. 60-67, 1967.

[11] J. M. Kleinberg, "Navigation in a small world," Nature, vol. 406, no. 6798, August 2000.

[12] J. Kleinberg, "The small-world phenomenon: An algorithmic perspective," in Proceedings of the 32nd ACM Symposium on Theory of Computing, 2000.

[13] J. M. Kleinberg, "Small-world phenomena and the dynamics of information," in Advances in Neural Information Processing Systems (NIPS) 14. MIT Press, 2001, p. 2001

[14] D. J. Watts, P. S. Dodds, and M. E. J. Newman, "Identity and search in social networks," Science, vol. 296, pp. 1302-1305, 2002.

[15] A. Hotho, R. Jäschke, C. Schmitz, and G. Stumme, "Bibsonomy: A social bookmark and publication sharing system," in Proceedings of the Conceptual Structures Tool Interoperability Workshop at the 14th International Conference on Conceptual Structures, 2006, pp. 87-102.

[16] T. Eda, T. Uchiyama, T. Uchiyama, and M. Yoshikawa, "Signaling emotion in tagclouds," in WWW '09: Proceedings of the 18th international conference on World wide web. New York, NY, USA: ACM, 2009, pp. $1199-1200$.
[17] O. Kaser and D. Lemire, "Tag-Cloud Drawing: Algorithms for Cloud Visualization," Proceedings of Tagging and Metadata for Social Information Organization (WWW 2007), 2007. [Online]. Available: http://arxiv.org/abs/cs/0703109v2

[18] J. Leskovec, J. Kleinberg, and C. Faloutsos, "Graphs over time: densification laws, shrinking diameters and possible explanations," in $K D D$ '05: Proceedings of the eleventh ACM SIGKDD international conference on Knowledge discovery in data mining. New York, NY, USA: ACM, 2005, pp. 177-187.

[19] L. A. Adamic, R. M. Lukose, A. R. Puniyani, and B. A. Huberman, "Search in power-law networks," Physical Review E, vol. 64, no. 4, pp. $0461351-8$, Sep 2001.

[20] Y. Zhang, B. Jansen, and A. Spink, "Time series analysis of a Web search engine transaction log," Information Processing \& Management, vol. 45, no. 2, pp. 230-245, 2009.

[21] P. Erdos and A. Renyi, "On the evolution of random graphs," Publ. Math. Inst. Hung. Acad. Sci, vol. 5, pp. 17-61, 1960.

[22] B. Bollobás and W. F. de la Vega, "The diameter of random regular graphs," Combinatorica, vol. 2, no. 2, pp. 125-134, 1982.

[23] B. Bollobás and F. R. K. Chung, "The diameter of a cycle plus a random matching," SIAM J. Discret. Math., vol. 1, no. 3, pp. 328-333, 1988.

[24] A. Hotho, R. Jäschke, C. Schmitz, and G. Stumme, "Folkrank: A ranking algorithm for folksonomies," in Proc. FGIR 2006, 2006.

[25] C. Koerner, D. Benz, A. Hotho, M. Strohmaier, and G. Stumme, "Stop thinking, start tagging: Tag semantics arise from collaborative verbosity," in 19th International World Wide Web Conference (WWW2010), Raleigh, NC, USA, April 26-30, ACM, 2010.

[26] J. Kleinfeld, "Could it be a big world after all? The six degrees of separation myth," Society, April 2002.

[27] M. Kochen, Ed., The Small World. Norwood, NJ: Ablex, 1989.

[28] D. J. Watts and S. H. Strogatz, "Collective dynamics of small-world networks," Nature, vol. 393, no. 6684, pp. 440-442, June 1998

[29] A. Dieberger, "Supporting social navigation on the world wide web," Int. J. Hum.-Comput. Stud., vol. 46, no. 6, pp. 805-825, 1997.

[30] D. Millen and J. Feinberg, "Using social tagging to improve social navigation," in Workshop on the Social Navigation and Community Based Adaptation Technologies. Dublin, Ireland. Citeseer, 2006. [Online]. Available: http://citeseerx.ist.psu.edu/viewdoc/download?doi= 10.1.1.92.5563 \\&amp;rep=rep $1 \backslash \& a m p ;$ type $=$ pdf

[31] M. Strohmaier, C. Trattner, and D. Helic, "The benefits and limitations of tag clouds as a tool for social navigation from a network-theoretic perspective," Journal of Universal Computer Science, 2010, (UNDER REVIEW).

[32] W.-T. Fu and P. Pirolli, "Snif-act: a cognitive model of user navigation on the world wide web," Hum.-Comput. Interact., vol. 22, no. 4, pp. $355-412,2007$.

[33] P. Pirolli, "An elementary social information foraging model," in Proceedings of the 27th international conference on Human factors in computing systems. ACM, 2009, pp. 605-614.

[34] M. Strohmaier, C. Koerner, and R. Kern, "Why do users tag? Detecting users' motivation for tagging in social tagging systems," in International AAAI Conference on Weblogs and Social Media (ICWSM2010), Washington, DC, USA, May 23-26, 2010.

[35] C. Koerner, R. Kern, H. P. Grahsl, and M. Strohmaier, "Of categorizers and describers: An evaluation of quantitative measures for tagging motivation," in 21st ACM SIGWEB Conference on Hypertext and Hypermedia (HT 2010), Toronto, Canada, ACM, Toronto, Canada, June 2010.

[36] E. H. Chi and T. Mytkowicz, "Understanding the efficiency of social tagging systems using information theory," in $H T$ '08: Proceedings of the nineteenth ACM conference on Hypertext and hypermedia. New York, NY, USA: ACM, 2008, pp. 81-88.

[37] R. Li, S. Bao, Y. Yu, B. Fei, and Z. Su, "Towards effective browsing of large scale social annotations," Proceedings of the 16th international conference on World Wide Web, p. 952, 2007. [Online]. Available: http://portal.acm.org/citation.cfm?id=1242700

[38] K. Aouiche, D. Lemire, and R. Godin, "Web 2.0 OLAP: From Data Cubes to Tag Clouds," 4th International Conference, WEBIST 2008, vol. 18, 2008. [Online]. Available: http://www.springerlink.com/index/ 10.1007/978-3-642-01344-7

[39] P. Heymann, A. Paepcke, and H. Garcia-Molina, "Tagging human knowledge," in Proceedings of the Third ACM International Conference on Web Search and Data Mining. New York, NY, USA: ACM, 2010, pp. 51-61. 